\title{
IN-ORBIT TREND ANALYSIS OF GALILEO SATELLITES FOR POWER SOURCES DEGRADATION ESTIMATION
}

\author{
Frederic Bard $^{(1)}$, Aurore Carré ${ }^{(1)}$, Pablo Fernandez ${ }^{(1)}$, Maria Nestoridi $^{(1)}$, Sergio Alia ${ }^{(2)}$, Aakesh Datta $^{(2)}$, \\ Olabisi Durodola ${ }^{(3)}$ \\ (1) European Space Agency - European Space Research and Technology Centre (ESTEC), The Netherlands, \\ Frederic.Bard@esa.int \\ (2) OHB System AG, Universitätalle 27, D-28359 Bremen, Germany, Sergio.Alia@ohb.de \\ (3) ADS GmbH, Robert-Koch Str.1,82024 Taufkirchen, Germany, Olabisi.Durodola2@airbus.com
}

\begin{abstract}
This paper presents the IOV and FOC Battery in orbit trend analysis and degradation modelling based on the Galileo experience in the frame of the ESPC 2016. Galileo provides a unique opportunity to study a constellation of satellites in the Medium Earth Orbit (MEO) to make a statistical analysis of power sources degradations due to its environment.
\end{abstract}

From a project point of view the objective of the study is to establish a process to obtain the in-orbit battery degradations. Accurate battery degradation knowledge will allow precise battery management from operation teams (battery fade strategy and combined Earth Lunar eclipse power estimations). Another key feature of this study is to be able to assess different battery performance (Saft and ABSL) under very similar mission requirements over a long period (electrical profile, temperature, and environment).

This paper reports on the first four years in orbit of IOV PFM satellite (10 eclipse seasons and still running) and on-going FOC satellite telemetry (TM) analysis (starting with two years of data from FOC GSAT201 and GSAT202).

The results are in all cases better than the predictions, which is expected due to the usage of conservatives assumptions in the design to cover (for both IOV and FOC) worst case scenario for the entire constellation. It should be noted that the FOC GSAT201 and GSAT202 batteries are degrading slightly faster than the 6 others FOC batteries identified GSAT203, GSAT204, GSAT205, GSAT206, GSAT208 and GSAT209, but still below predictions due to their peculiar unexpected orbit reached after launch (higher DoD up to $42 \%$ measured due to longer eclipses). These 2 satellites will require specific degradation monitoring.

\section{INTRODUCTION}

The Galileo satellites compose a constellation in the MEO Orbit. It is composed of 24 reference satellites on 3 orbital planes separated by $120^{\circ}$ right ascension of the ascending node (Plane A, B and C). Galileo is on a circular orbit of $29600 \mathrm{~km}$, at an inclination of $56^{\circ}$. The satellites design lifetime is 12 years.
The battery model correlation consist of tuning the ageing factor that lead to the cell degradations. Applying this analysis on all the batteries of the constellation, the trend analysis is obtained as well as a comparison in performance of the different battery types used on-board Galileo Satellites.

\section{GALILEO POWER SUBSYSTEM}

The satellite specification remaining unchanged for both projects, both IOV and FOC power subsystem are built on a $50 \mathrm{~V}$ fully regulated bus. The nominal spacecraft power is in the order of $1550 \mathrm{~W}$ based on conservative power budget with a very stable payload consumption. Both spacecraft designs use the same solar array provided by Airbus NL capable of producing more than $1904 \mathrm{~W}$ at EOL (prediction assuming worst case degradations and 1 section failed). Each spacecraft are using Sequential Switching Shunt Regulators (S3R) to connect to the SA sections. The Solar array design, based on GaAs triple junction $28 \%$ cells, is made of 29 cells per strings for a total of 88 strings. Thanks to a very stable solar array pointing law (solar array always normal to the sun) the operating temperature remains between 60 and $63^{\circ} \mathrm{C}$ (outside of eclipse) and the power produced is affected only by the seasonal variations and radiation degradation.

The batteries however are coming from two different manufacturers: IOV is equipped with Saft VES180 and VES140 cells and FOC is equipped with ABSL Sony $18650 \mathrm{HC}$ cells and $18650 \mathrm{HCM}$ cells.

This MEO orbit has a period of 14.08 hours with two Earth eclipse seasons per year. The eclipses are presented in Fig. 2 with a maximum duration of $60 \mathrm{~min}$. 


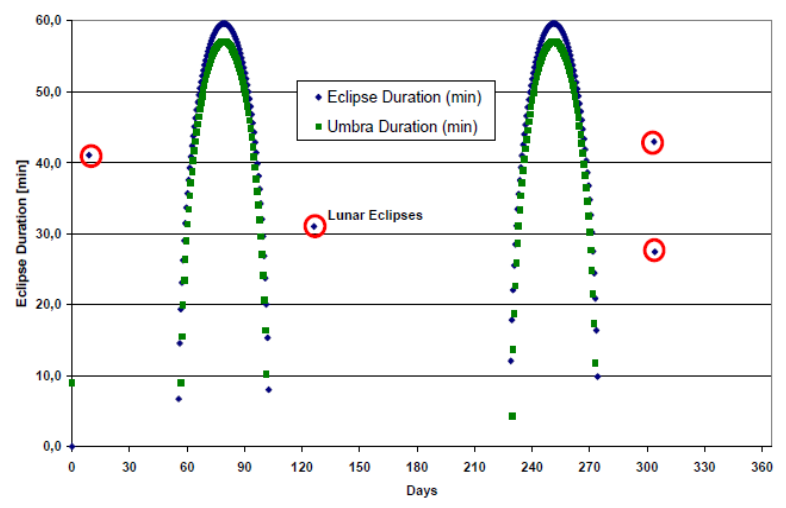

Figure 1. Galileo orbit Earth eclipse profile

During its lifetime, Galileo batteries will endure a maximum of 2064 eclipses. This is significantly more than for typical GEO satellites (1080 cycles over 12 years). However it should be noted that due to the specificities of each orbital planes (which has a small RAAN drift of about -10 degrees per year), the eclipses seasons last between 25 and 47 days. This means that depending on the Sun, Earth and orbital plane alignment the number of eclipses per season could vary between 45 cycles and 86 cycles.

It is worth mentioning that, due to a wrong orbit injection from Soyuz-Fregat in August 2014, the FOC GSAT201 and GSAT202 have reached an elliptical orbit (resonant orbit) with Earth eclipses lasting up to $68 \mathrm{~min}$.

This study will establish the actual degradation impact due to higher DoD on these two satellites.

\section{IOV BATTERY MODELLING}

The IOV batteries have been provided by Saft. Based on a P-S topology, they are composed of 9 cell-packs serial connected. Each cell-pack consists in 3 cells parallel connected, using the VES180 cell on GSAT101 and GSAT102 and the VES140 cell on GSAT103 and GSAT104.

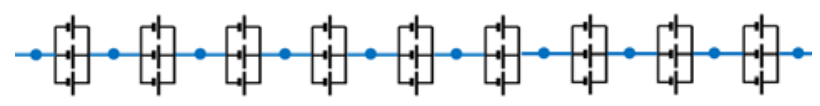

Figure 2. 3P9S IOV battery configuration

3P9S VES180 batteries have a nameplate energy of $4900 \mathrm{Wh}$, and the VES140 have a nameplate energy of $3585 \mathrm{Wh}$. The Saft batteries include a passive balancing system optimizing the cell voltages over lifetime and a by-pass system allowing to isolate a failed cell.

The batteries are controlled in temperature to a value of $18.5^{\circ} \mathrm{C}$ in average for the 12 years of lifetime. IOV predictions are compared to measured data, considering a Used Energy of $45 \%$ in average with respect to the EOL energy.
IOV batteries telemetry have been acquired and postprocessed. Using the current and temperature profiles during the longest Earth Eclipses and applying this profile into on-ground simulation tools, the battery degradation parameters could be obtained. Fig. 3 shows a view of the IOV battery module

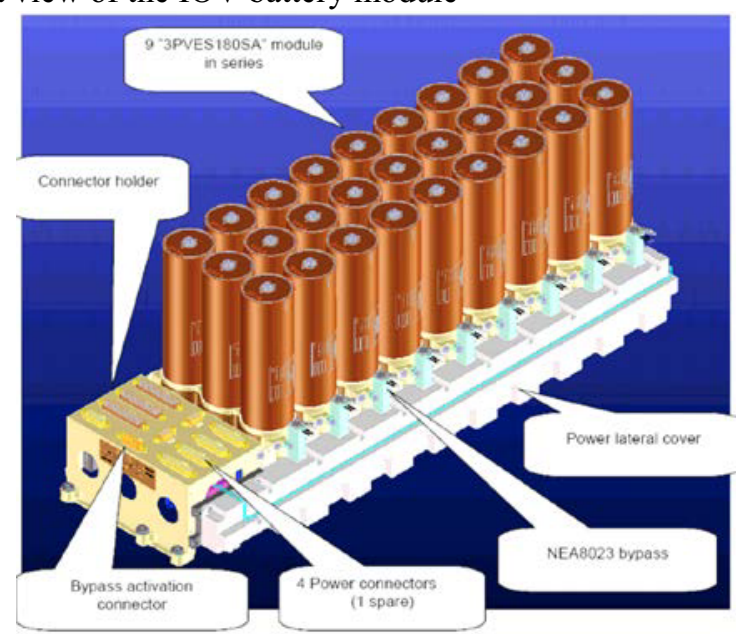

Figure 3. Galileo 3P9S IOV battery configuration

\subsection{Inputs and assumptions}

The simulations were run for satellite IOV PFM (FM02 battery based on VES180 cell). Table 1 presents the summary of the IOV batteries for both IOV PFM and FM02 satellites. The latest eclipses season was used to establish the latest degradations figures of the batteries.

Table 1. Galileo IOV batteries summary table

\begin{tabular}{|c|c|c|}
\hline S/C & IOV PFM & IOV FM02 \\
\hline LEOP Name & 3A5 & 3A6 \\
\hline Sat Name & GSAT101 & GSAT102 \\
\hline battery type & VES180 & VES180 \\
\hline Battery name & FM02 & EQM \\
\hline Plane & B & B \\
\hline Launch date & $21 / 10 / 2011$ & $21 / 10 / 2011$ \\
\hline Eclipse Se ason 1 & Jan-12 & Jan-12 \\
\hline Eclipse Se ason 2 & Jur 12 & Jul-12 \\
\hline Eclipse Se ason 3 & Jan-13 & Jan-13 \\
\hline Eclipse Se ason 4 & Jut13 & Jul-13 \\
\hline Eclipse Season 5 & Jan-14 & Jan-14 \\
\hline Eclipse Season 6 & Jut14 & Jul-14 \\
\hline Eclipse Season 7 & Jan-15 & Jan-15 \\
\hline Eclipse Season 8 & Jut15 & Jul-15 \\
\hline Eclipse Season 9 & 05/01/2016 & $26 / 12 / 2016$ \\
\hline Eclipse Se ason 10 & $27 / 06 / 2016$ & $29 / 06 / 2016$ \\
\hline
\end{tabular}

The following hypotheses were considered for the IOV GSAT101 (FM02 battery):

- Battery characteristics provided by Saft during final on-ground testing (energy, internal resistance)

- 581 days on-ground storage at $20^{\circ} \mathrm{C}$ including 10 cycles at $100 \%$ DOD

- $\quad$ LEOP power consumption of $201 \mathrm{Wh}$, at $18^{\circ} \mathrm{C}$, 
with a total duration about 5.8 hours (to be compared to the $4900 \mathrm{Wh}$ used at worst case dimensioning for the battery sizing)

- The MEO mission profile considering a S/C power consumption of $1286 \mathrm{~W}$ in average (to be compared to the $1562 \mathrm{~W}$ used as worst case dimensioning for the design)

- The battery temperature at $18^{\circ} \mathrm{C}$ during both eclipse and solstice periods

- The number of eclipses cycles identified on Plane B during the latest eclipse season is only 46 cycles instead of 86

The following plot provides an example of an eclipse season profile on the IOV FM02 battery (on-board GSAT101).

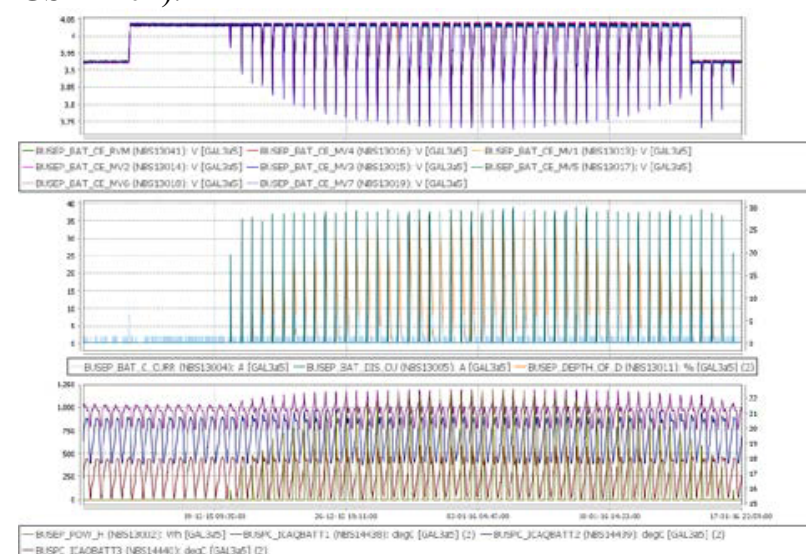

Figure 4. IOV FM02 battery cycling during eclipse season 9

The battery management strategy is presented in Fig. 4. At the end of the eclipse phase, the cell packs voltages are reduced to a voltage of $3.92 \mathrm{~V}$ ensuring a lower SoC during sun phase to increase the lifetime.

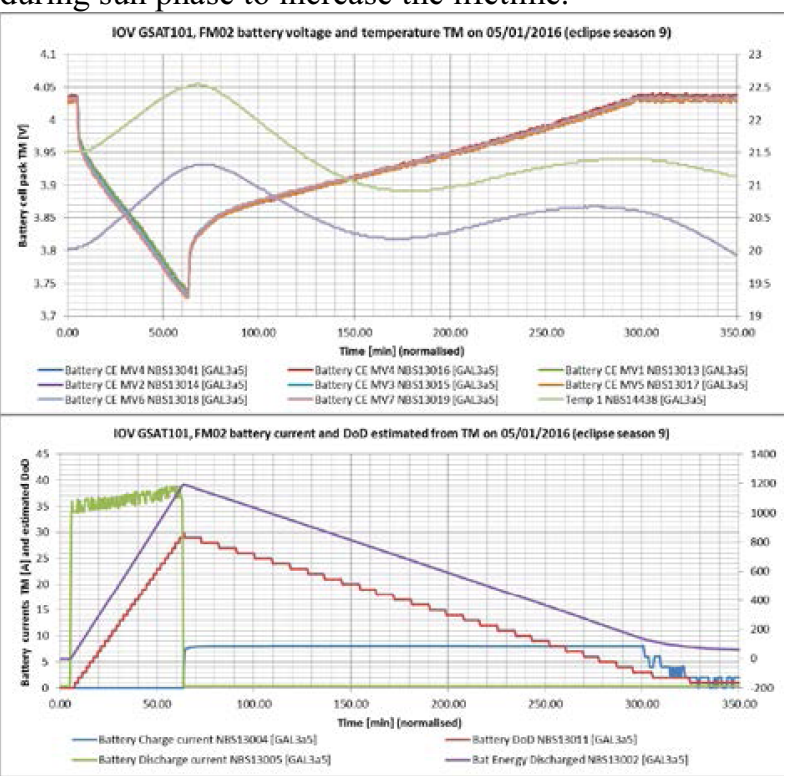

Figure 5. IOV FM02 battery telemetry during sun to eclipse transition in eclipse season 9
Figure 5. shows the transition from sun to eclipse. It could be seen that the dispersion of the cell packs are very limited at this stage.

\subsection{Simulation results}

For IOV batteries, the correlation is based on the SLIM simulation tool provided by Saft. SLIM (Saft Space Liion Model) is a macroscopic electrochemical model developed since 2005. The purpose of this model is to calculate the EOL performances of Saft VES batteries taking into account the battery characteristics (acceptance energy and internal resistance), the specification or the mission profile and the in orbit battery management.

This model is a battery design tool vs satellite mission profile and for the IOV satellites, simulations have been done by using a MEO specific environment (orbital period, number of cycles, charge rate...).

Fig. 6 presents the simulation results (SLIM outputs) compared to the battery voltage telemetries (at battery level). It is worth noting that available voltage telemetries were at cells pack level. Thus, the battery voltage telemetry has been simply calculated by adding them and do not take into account any potential voltage drop due to ohmic losses link to cables. Consequently, the current difference between the telemetries and the predicted voltages are considered conservative and further investigations in this study will be done to fine tune this aspect and lead to more realistic outputs.

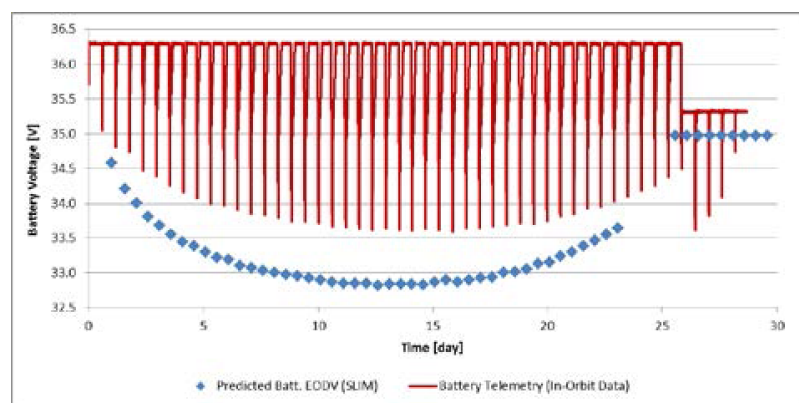

Figure 6. IOV FM02 battery EoD voltage comparison between SLIM and telemetry during eclipse season 9

Considering a single worst case eclipse of $60 \mathrm{~min}$, the SLIM output is compared with the battery voltage at battery level in Fig. 7. 


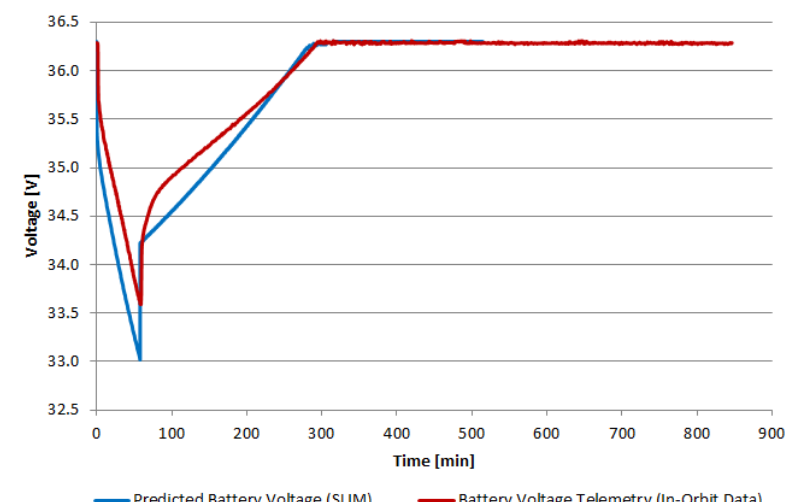

Figure 7. IOV GSAT101 battery voltage comparison during the longest eclipse (cycle 28) of season 9

The correlation between the battery voltage TM from IOV FM02 battery compared to the SLIM simulation results is confirming the fact that the IOV FM02 battery is behaving better than predicted. This is in line with the real in-orbit conditions (lower on-ground storage period, lower power consumption, lower number of eclipse cycles).

The predicted degradation over the complete mission was expected at $1.5 \%$. With the revised analysis performed after 9 eclipse seasons and extrapolated for the lifetime, the degradation figure is expected at $0.6 \%$. This is an additional margin which could be used for potential mission extension over the 12 years guaranteed of the IOV satellites.

\subsection{Conclusions and remaining activities}

The results obtained from the SLIM correlation exercise on IOV FM02 battery confirm that the battery is behaving well. All battery operations are in line with expected value such as :

- The charge and discharge management

- The temperatures range

- The balancing operations

Comparable analysis have already been performed on in-orbit behaviour of VES batteries from Saft (for instance [1] and [2]). This study is in line with these. As already stated, the study will be further processed. Indeed, investigations will be done to fine tune the hypotheses regarding the battery voltage acquisition.

\section{FOC BATTERY MODELLING AND TELEMETRY ANALYSIS}

Each FOC battery is comprised of one module, which contains 64 strings in parallel, each consisting of 11 cells in series. The design difference between IOV, i.e.Saft batteries and FOC, i.e. ABSL batteries is that the ABSL batteries do not contain a balancing system. The latter is feasible due to the highly uniform COTS cells and ABSL's proprietary screening and matching routine. [3]
The following figure presents the FOC battery provided by ABSL. It is based on the typical Sony $18650 \mathrm{HC}$ cell assembled into 4 blocks in a double deck battery module.

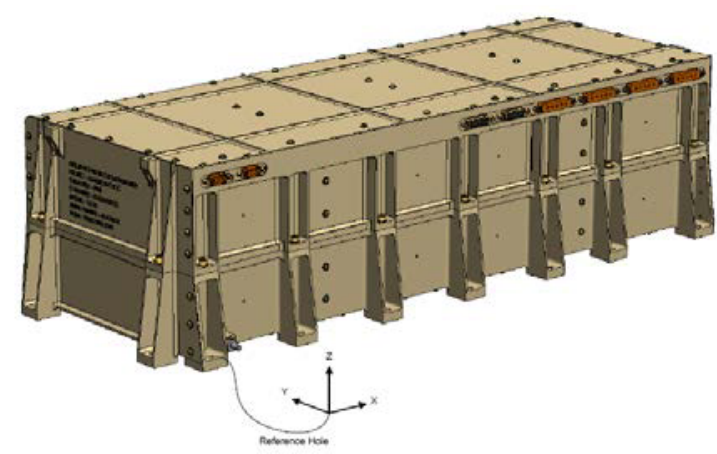

Figure 8. Galileo FOC battery from ABSL

\subsection{Inputs and assumptions}

The 11S64P nameplate energy is $3800 \mathrm{Wh}$. In Fig. 10, the predicted degradations over life time considering 4 year of storage and AIT activities followed by 12 years in orbit is presented for different solstice conditions.

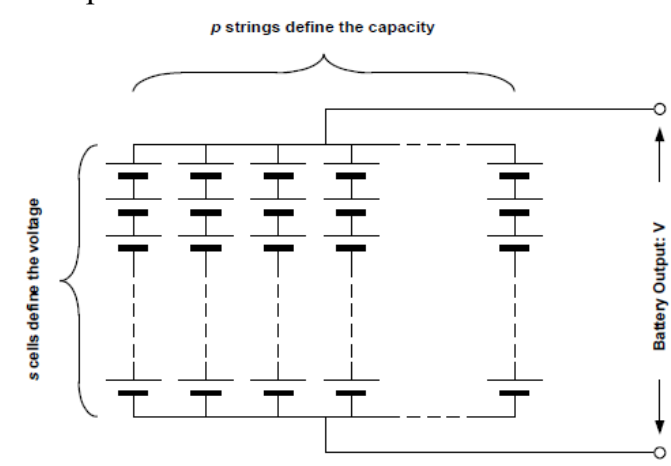

Figure 9. 11S64P FOC battery configuration

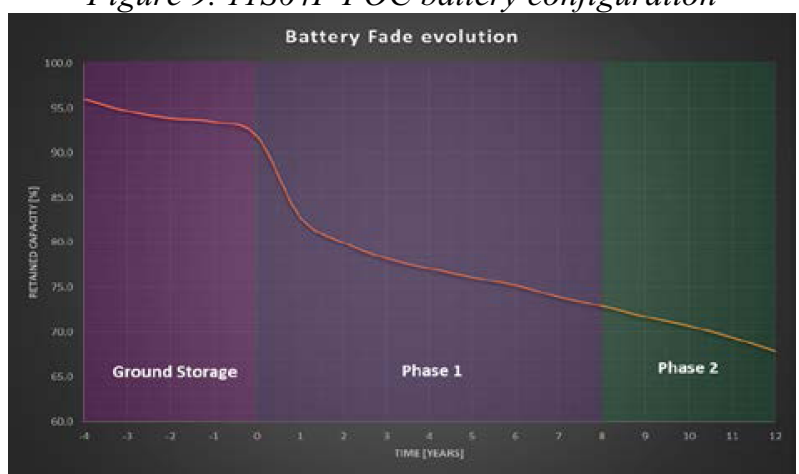

Figure 10. FOC battery degradation predicted based on LIFE model provided by ABSL considering a battery fade strategy implementation

Considering the nominal mission with the above constraints, the degradation are estimated at $32 \%$. This estimation is considering an average DoD of $35 \%$ during eclipse seasons ( 2 seasons per year) and a SoC of $80 \%$ during the solstice season. To be noted in the current predictions, the knee of the degradation curve is expected after 14 years in orbit after which the battery 
performances could not be guaranteed anymore. However the current results confirms that this inflection is to be expected later (allowing potential mission extension).

Fig. 11 presents the battery voltage evolution over the seasons by superposing 3 eclipses profiles of GSAT203. The first information is a clear decrease of the minimum voltage reached during the typical eclipse (60min) confirming a small increase in delta DoD from $27.2 \%$ during the first eclipse season up to $32 \%$ after one year in orbit (stabilised during the 2 last eclipse seasons).

The battery management approach is also visible with a reduced EoC voltage at $45.2 \mathrm{~V}$ applicable for the first 8 years in orbit to increase the battery lifetime.

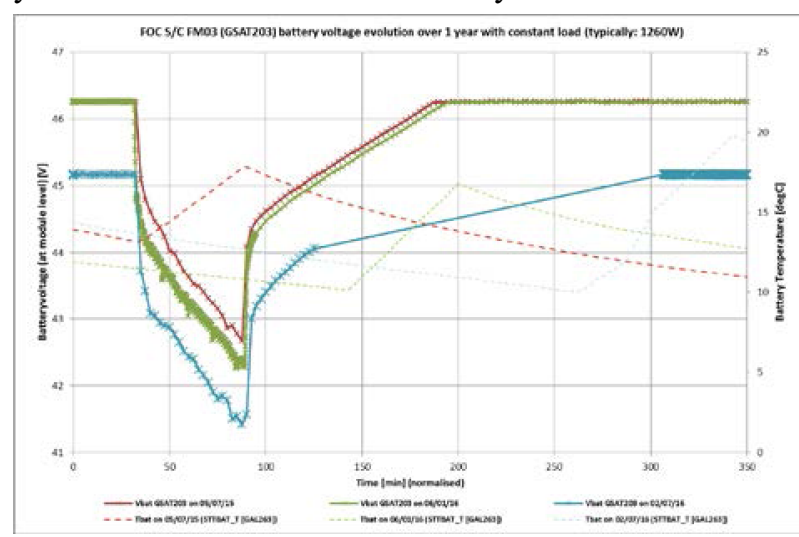

Figure 11. FOC FM03 battery telemetry during sun to eclipse transition over two orbits.

Based on the telemetry data the FOC spacecraft consumptions could be established with an accuracy of less than $3 \%$. The average power consumption for the FOC constellation is:

- In nominal mode: between $1250 \mathrm{~W}$ and $1300 \mathrm{~W}$

- In safe mode: between 900W and 930W

All the telemetries obtained from the in-orbit spacecraft fall into this range (see Table 2).

It is worth to compare these data with the power of $1550 \mathrm{~W}$ which was used at the time of the design phase for battery lifetime predictions.

\subsection{Results and main conclusions}

A total of 17 simulations were run for the FOC batteries for a total of 8 spacecraft in nominal operations over the last 2 years.

As a reference case GSAT203 (FOC FM03) will be used to illustrate the methodology of the model correlation. BEAST models are feed with in-orbit telemetry of the battery current and temperatures during the discharge and charge phase, then the Beast simulation voltage is adjusted to the in-orbit battery voltage applying the battery capacity degradation coefficient and the internal resistance increase. This is an iterative process until the simulation voltage match as best as possible the telemetry. This results in estimated degradation figures which could be compared to the predictions made at the design phase. All results are provided in Table 2. Figures 12, 13 and 14 illustrate the correlation of the battery in-orbit voltage to the Beast simulation voltage.

The matching is not perfect due to the EMF jumps at the transitions between charge and discharge.

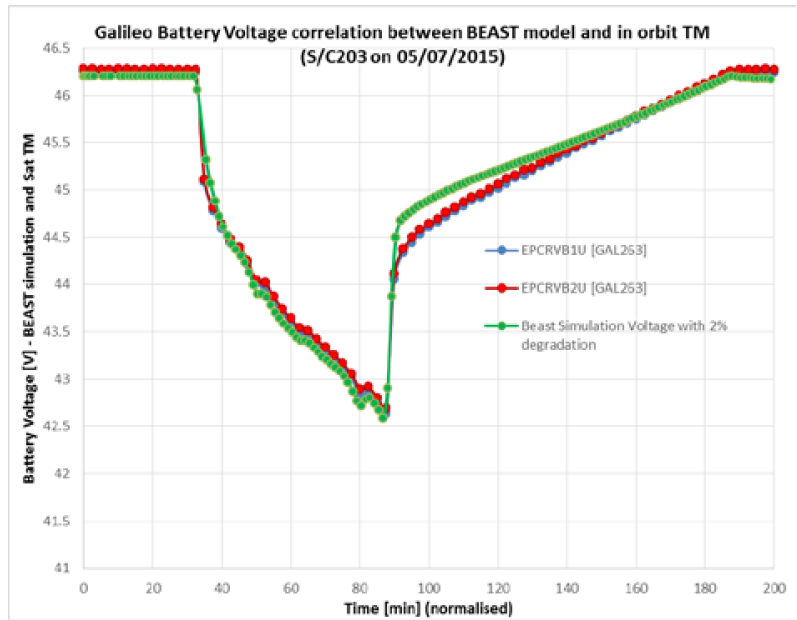

Figure 12. FOC GSAT203 battery voltage correlation for the reference eclipse on 05/07/2015

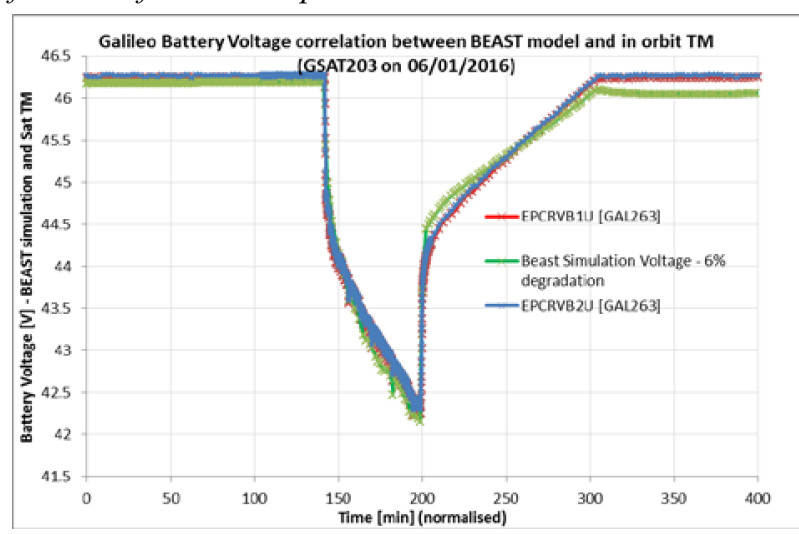

Figure 13. FOC GSAT203 battery voltage correlation for the reference eclipse on 06/01/2016

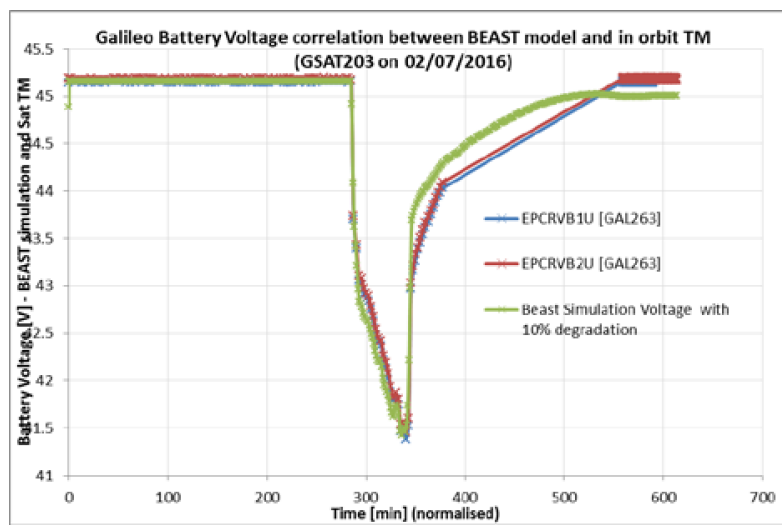

Figure 14. FOC GSAT203 battery voltage correlation for the reference eclipse on 02/07/2016 
Table 2. Summary of FOC battery degradations

\begin{tabular}{|c|c|c|c|c|c|c|c|c|c|}
\hline s/c & Launch Date & $\begin{array}{c}\text { Reference Eclipse } \\
\text { Date }\end{array}$ & $\begin{array}{l}\text { Difference } \\
\text { (months) }\end{array}$ & $\begin{array}{c}\text { Fade Expected } \\
{[\%]}\end{array}$ & $\begin{array}{c}\text { Fade Beast } \\
\text { Simulation [\%] }\end{array}$ & $\begin{array}{l}\text { Resistance } \\
\text { increase } \\
\text { Expected[\%] }\end{array}$ & $\begin{array}{c}\text { Resistance } \\
\text { increase Beast } \\
\text { simulation [\%] }\end{array}$ & $\begin{array}{c}\text { Power } \\
\text { consumption [W] }\end{array}$ & Delta DoD [\%] \\
\hline \multirow{2}{*}{ GSAT201 } & $22 / 08 / 2014$ & $24 / 06 / 2015$ & 10 & 18.8 & 7 & 21.8 & 9 & 1298 & $36.20 \%$ \\
\hline & $22 / 08 / 2014$ & $16 / 06 / 2016$ & 22 & 21.4 & 15 & 29.9 & 20 & 1249 & $39.50 \%$ \\
\hline \multirow{2}{*}{ GSAT202 } & $22 / 08 / 2014$ & $28 / 06 / 2015$ & 10 & 18.8 & 7 & 21.8 & 9 & 1265 & $36.70 \%$ \\
\hline & $22 / 08 / 2014$ & $12 / 06 / 2016$ & 22 & 21.4 & 18 & 29.9 & 25 & 1284 & $42.00 \%$ \\
\hline \multirow{3}{*}{ GSAT203 } & $27 / 03 / 2015$ & $05 / 07 / 2015$ & 4 & 13.45 & 2 & 17.75 & 5 & 1255 & $27.20 \%$ \\
\hline & $27 / 03 / 2015$ & $06 / 01 / 2016$ & 10 & 18.8 & 6 & 21.8 & 10 & 1260 & $31.70 \%$ \\
\hline & $27 / 03 / 2015$ & $02 / 07 / 2016$ & 16 & 20.1 & 10 & 25.85 & 15 & 1264 & $32.10 \%$ \\
\hline \multirow{3}{*}{ GSAT204 } & $27 / 03 / 2015$ & $03 / 07 / 2015$ & 4 & 13.45 & 3 & 17.75 & 4 & 932 & $\mathrm{~N} / \mathrm{A}$ \\
\hline & $27 / 03 / 2015$ & $01 / 01 / 2016$ & 10 & 18.8 & 8 & 21.8 & 10 & 1283 & $31.40 \%$ \\
\hline & $27 / 03 / 2015$ & $29 / 06 / 2016$ & 15 & 20.1 & 10 & 25.85 & 10 & 1305 & $34.10 \%$ \\
\hline \multirow{2}{*}{ GSAT205 } & $11 / 09 / 2015$ & $01 / 02 / 2016$ & 5 & 13.45 & 4 & 17.75 & 5 & 1271 & $31.50 \%$ \\
\hline & $11 / 09 / 2015$ & $18 / 07 / 2016$ & 10 & 18.8 & 7 & 21.8 & 5 & 1259 & $27.50 \%$ \\
\hline \multirow{2}{*}{ GSAT206 } & $11 / 09 / 2015$ & $01 / 02 / 2016$ & 5 & 13.45 & 3 & 17.75 & 4 & 1268 & $29.75 \%$ \\
\hline & $11 / 09 / 2015$ & $23 / 07 / 2016$ & 10 & 18.8 & 8 & 21.8 & 4 & 1294.8 & $32.10 \%$ \\
\hline GSAT208 & $17 / 12 / 2015$ & $07 / 04 / 2016$ & 4 & 13.45 & 2 & 17.75 & 3 & 1318 & $31 \%$ \\
\hline GSAT209 & $17 / 12 / 2015$ & $07 / 04 / 2016$ & 4 & 13.45 & 2 & 17.75 & 3 & 1303 & $31.20 \%$ \\
\hline
\end{tabular}

Based on Table 2, the following conclusions could be drawn:

- Table 2 presents the capacity fade obtained estimated based on TM analysis compared to the predictions (fade expected in reference to figure 10).

- The average DoD for all $\mathrm{S} / \mathrm{Cs}$ is in the order of $27 \%$ to $32 \%$, with the noticeable exception of GSAT201 and GSAT202 where the maximum average DoD reached $42 \%$

- For the $\mathrm{S} / \mathrm{C}$ in the nominal orbit, the correlation exercise highlighted a significant margin compared to the life predications. This is due to the conservative assumptions made in the design phase in terms of $\mathrm{S} / \mathrm{C}$ power consumption (in average the $\mathrm{S} / \mathrm{C}$ consumed $250 \mathrm{~W}$ less than predicted) and to a shorter onground AIT phase

- The correlation of the battery TM from GSAT201 and GSAT202 highlight a faster degradation (still within prediction levels), which is in-line with a longer eclipse of up to $68 \mathrm{~min}$ on the resonant orbit.

- At this stage of the analysis, no results were out of family indicating that none of the battery had suffered damages

The results confirmed the good behaviour of Galileo FOC batteries fleet, but this analysis is only the beginning and should be followed over longer time.

GSAT201 and GSAT202 batteries will require a specific monitoring to follow the degradation evolution. At this stage the end of life of the FOC batteries could not be assessed accurately, this will be done at a later stage following the progression over several years.

\section{CONCLUSIONS AND FUTURE WORK}

This study provides a quantitative data on the in-orbit performance of the Galileo satellite batteries which could be used for in-orbit trend analysis. All telemetries and correlation results acquired at this stage are showing better than predicted performances due to conservative assumptions made in the design phase.

For IOV, only the FM02 battery correlation has been established at this stage, but all in-orbit telemetries acquired on all IOV spacecraft are showing normal behaviour in voltage, current and temperature.

Future work on the Galileo battery in-orbit analysis will involve extension of the correlation exercise to all IOV spacecraft (IOV GSAT102 which include VES180 EQM module, IOV GSAT103 including PFM03 VES140 and IOV GSAT104 including FM04 VES140).

Furthermore the battery model provided by BEAST is sufficient for a first order estimation of the battery degradation but is conservative in terms of the EMF jumps during charge and discharge transitions. To model accurately these effects it is necessary to use a transient model for the battery including capacitive effects (diffusion effect). This activity will be performed using more refined models in the second step of the Galileo in-orbit TM assessment.

\section{REFERENCES}

1. Borthomieu Y., Gambini D. Saft Li-Ion VES140S battery flight experience return on TAS Space Bus platforms, ESPC 2014

2. Borthomieu Y., Prevot D., Massot J., Tastet P., Simon E. VES100/140 lithium-ion cells LEO lifetest results \& Proteus flight heritage, ESPC 2011

3. Fredon S., Melac L., Simmons N., Long duration LEO mission experience with ABSL batteries onboard the myriad smallsat platform, ESPC 2011. 